\title{
Fuentes de Conocimiento en Empresas Biotecnológicas
}

\author{
Viridiana Núñez López
}

Resumen: Los resultados empíricos respecto a cómo las empresas obtienen el conocimiento externo, sobre todo en sectores intensivos en conocimiento, no han sido concluyentes. Mientras que la mayoría de los estudios previos considera para el análisis un mecanismo de acceso al conocimiento, el presente estudio considera tres mecanismos: relaciones formales e informales y movilidad laboral. El estudio es cualitativo, exploratorio, e intenta identificar cuáles son las fuentes de conocimiento externo de las empresas biotecnológicas localizadas en la región occidente de México. Los resultados muestran que más de la mitad de los vínculos para obtener el conocimiento se establecen a nivel regional, predominando las relaciones formales. Sin embargo, los resultados sugieren que es el conocimiento adquirido a nivel internacional el que confiere a la empresa una competitividad de clase mundial.

Palabras clave: conocimiento externo; aprendizaje; empresa biotecnológica; país emergente.

Abstract: Empirical results regarding how the firms obtain external knowledge, mainly firms belonging to intensive-knowledge sectors, have been inconclusive. While the majority of early studies considered only one mechanism of knowledge access, in the current study three mechanisms are considered: formal and informal relationships, and laboral mobility. This study is qualitative, exploratory and attempts to identify external knowledge sources in the biotechnology firms located in the occidental region of Mexico. The results show that more than half of the links for getting the knowledge are at the regional level. However, the results suggest that the knowledge acquired at the international level confers on a world-class competitiveness to the firm.

Keywords: External knowledge; learning; biotechnology firm; emerging economy.

Submitted August 26th 2015 / Approved September 30th 2015

\section{Introducción}

A diferencia de otros sectores, la industria biotecnológica es relativamente reciente (Powell, Koput, y Smith, 1996). Por ejemplo, la industria química posee una larga historia de investigación científica básica $^{1}$, asimismo el conocimiento teórico ya ha sido codificado (en revistas científicas, libros de texto), es decir, es un campo maduro. La búsqueda y selección de procesos alternativos se realiza a partir de una serie de principios, leyes y modelos ya dados, que describen las relaciones entre variables ${ }^{2}$. El sector biotecnológico se encuentra en una etapa muy diferente. La primera empresa fue fundada en la década de los 70. La investigación básica sobre ingeniería de procesos biotecnológicos a gran escala ha sido poca, por lo que las variables críticas pueden ser desconocidas, haciendo difícil predecir cómo los procesos que han sido probados en el laboratorio se comportarán en la fábrica (Pisano, 1994).

Inmersa en la economía del conocimiento, la biotecnología es intensiva en conocimiento (Decarolis y Deeds, 1999; Moen, 2001;
Powell et al., 1996; Prevezer, 1997; Salavisa, Sousa y Fontes, 2012) complejo y en expansión (Powell, 1998; Rank, Rank y Wald, 2006). La empresa precisa del conocimiento externo que se genera en universidades, instituciones e incluso otras empresas (Audretsch y Feldman, 2003; Decarolis y Deeds, 1999; Khoury y Pleggenkuhle, 2011; Powell et al., 1996).

La combinación de conocimiento permite llevar a cabo: i) Innovación radical (Doloreux, 2002; Grandstrand, 1998; Salavisa et al., 2012; Song, Almeida y Wu, 2003), siendo esta habilidad la que puede determinar la sobrevivencia (Owen-Smith y Powell, 2004) o competitividad (Smith, 2000) de la empresa. ii) Innovación incremental, la cual se refleja en la mejora de productos y procesos. iii) Realización de procesos (Decarolis y Deeds, 1999; Moen, 2001; Prevezer, 1997; Salavisa et al., 2012). Además, el conocimiento conduce al aprendizaje. La conexión entre ambas variables radica en que el aprendizaje conlleva cambios en la base de conocimiento (Chaminade y Edquist, 2005; Doloreux, 2002; Smith, 2000). La importancia del aprendizaje radica en que es la fuente de las habilidades y capacidades que posee la empresa (Pisano, 1994).

*Departamento de Ciencias Sociales y Jurídicas, Universidad de Guadalajara, Jalisco, México, E-mail: viri_nunez@yahoo.com.mx

(1) La industria química remonta sus orígenes al siglo XVIII. La producción de fármacos, utilizando síntesis química, ha tenido lugar desde finales del siglo XIX (Pisano, 1994).

(2) Como presión, volumen y temperatura.

ISSN: 0718-2724. (http://jotmi.org)

Journal of Technology Management \& Innovation @ Universidad Alberto Hurtado, Facultad de Economía y Negocios. 
Los mecanismos para acceder al conocimiento externo están basados en la interacción social (Doloreux y Parto, 2005; Larsson et al., 1998), ejemplos de ellos son: la relación formal e informal, la movilidad laboral. No obstante, los resultados no han sido concluyentes respecto a la manera en que la empresa obtiene el conocimiento externo. La literatura empírica se ha enfocado al estudio de un mecanismo de acceso a conocimiento externo ${ }^{3}$ a la vez, por lo que se pierde de vista el entorno bajo el que se están dando estos resultados;. Segundo, es frecuente realizar investigaciones con datos secundarios, con sus consecuentes limitaciones ${ }^{4}$. Tercero, los estudios se han enfocado a países desarrollados.

El presente estudio intenta identificar cuáles son los mecanismos de conocimiento externo que utiliza la empresa como parte de su proceso de aprendizaje. Para ello considera tres mecanismos, utiliza datos primarios y se aplicó a empresas biotecnológicas del occidente de México. Los resultados muestran que el principal mecanismo de acceso a conocimiento externo es la relación formal a nivel regional. Sin embargo, los lazos (o vínculos) para acceder al conocimiento no están construidos del todo, sino que se van formando a medida que surgen las necesidades de conocimiento, pero también los obstáculos.

El presente artículo está organizado de la siguiente manera: la segunda sección expone el marco analítico en que se basa el estudio. La tercera sección describe la estrategia metodológica aplicada en la investigación así como los datos utilizados. La cuarta sección presenta los resultados obtenidos. La última sección está dedicada a la discusión.

\section{Marco analítico}

Algunos tipos de interacción social abordados en la literatura son: alianzas (Carayannopoulos y Auster, 2010; Haeussler, Patzelt y Zahra, 2012; Khoury y Pleggenkuhle, 2011; Larsson et al., 1998); redes (Breschi y Catalini, 2010; Fabrizio, 2009; Porter et al., 1996; Salavisa et al., 2012); colaboraciones (Powell et al., 1996). En otras palabras, la interacción social puede darse a través de relaciones formales o informales, siendo esta clasificación la que considera la presente investigación.

Definimos relación formal como un "acuerdo formal/codificado entre los actores, generalmente involucra un sistema de autoridad, distribución de competencias, derechos y deberes y un dispositivo de resolución de conflictos" (Salavisa et al., 2012, p. 388); colaboración informal como aquella "asociada con lazos personales que son directamente movilizados o actúan como mediadores en el acceso a los recursos" (Salavisa et al., 2012, p. 388). Al entablarse una relación formal o informal se crea un lazo (o vínculo) entre los participantes.
Empíricamente la movilidad laboral se ha tratado desde diferentes perspectivas. En este trabajo consideramos que la empresa a la que se mueve el empleado se ve beneficiada por la derrama de conocimiento, siendo la movilidad una manera de tener acceso al conocimiento externo. Definimos movilidad como el movimiento de trabajadores de una empresa a otra (Maliranta, Mohnen y Rouvinen, 2009), siempre y cuando el movimiento sea entre empresas biotecnológicas. Sin embargo, no en todas las aglomeraciones de empresas existe movilidad en una escala significativa. Por ejemplo, Lawton-Smith y Waters (2005) encuentran que la movilidad en Cambridge y Oxford, regiones que concentran industria de alta tecnología en Inglaterra, la movilidad no es mayor comparada con el resto del país.

En la presente investigación consideramos dos tipos de conocimiento: i) Conocimiento científico biotecnológico, denominado en este trabajo por simplicidad "conocimiento biotecnológico". ii) Conocimiento científico diferente al biotecnológico, es todo aquel conocimiento científico que no es biotecnológico pero que es útil a la empresa porque es complementario del área empresarial, denominado en este trabajo "conocimiento complementario".

El conocimiento es difícil de medir o evaluar (Audretsch y Feldman, 2003; Granstrand, 1998). Por ello, conocer el conjunto de lazos (que se establecen a partir de las relaciones formales e informales) para acceder a conocimiento externo es útil porque da cuenta de un flujo de conocimiento (Breschi y Catalini, 2010) el cual forma parte del proceso de aprendizaje ${ }^{5}$ de la empresa; además permitirá el diseño de instrumentos de política para apoyarlos (Balzat y Hanusch, 2004; Chaminade y Edquist, 2005; Doloreux, 2002; Edgington, 2008; Smith, 2000).

El presente estudio se inserta en este cuerpo de literatura. Intenta identificar las fuentes de conocimiento, esto es, el conjunto de lazos, formales e informales, de las empresas biotecnológicas de la región occidente de México en su búsqueda deliberada de conocimiento externo. Asimismo el estudio se ocupa de otro mecanismo de acceso a conocimiento, la movilidad laboral.

\section{Metodología}

De acuerdo con la OCDE (2009) ${ }^{6}$ en México existen varias zonas biotecnológicas (ver figura 1), una de ellas se ubica en la región occidente, que es en la que se centra la presente investigación.

De acuerdo a nuestra revisión, no encontramos una fuente que concentrara los datos sobre las empresas biotecnológicas localizadas en la región occidente, en parte, porque las empresas suelen agruparse por el giro industrial, y no por la técnica de producción que utilizan.

(3) Una excepción es el estudio de Tzabbar, Aharonson y Amburgey (2013) que consideran al mismo tiempo las colaboraciones formales y la movilidad.

(4) Por ejemplo, hay estudios que utilizan datos de patentes para definir que hubo flujo de conocimiento. Sin embargo, no todas las relaciones derivan en patentes porque no todas las invenciones son patentables o se patentan (Griliches, 1990), aunque sí haya una transmisión de conocimiento.

(5) Dado que el conocimiento conlleva cambios en la base de conocimiento de la empresa (Chaminade y Edquist, 2005; Doloreux, 2002; Smith, 2000) es inevitable hablar de aprendizaje, sobre todo cuando la empresa está haciendo esfuerzos deliberados por allegarse de conocimiento externo.

(6) El estudio de la OCDE identifica las regiones biotecnológicas en el mundo considerando tres niveles dados por el número de patentes en biotecnología. En México identifica varias regiones biotecnológicas, situadas todas, en la escala más baja (de 1 a 100 patentes). 
Derivado de nuestria búsqueda encontramos que existen 12 empresas biotecnológicas, el giro industrial se muestra en la figura 2 y la caracterización en la tabla 1. Por motivos de confidencialidad no se presenta el nombre real de la empresa, sino uno ficticio.
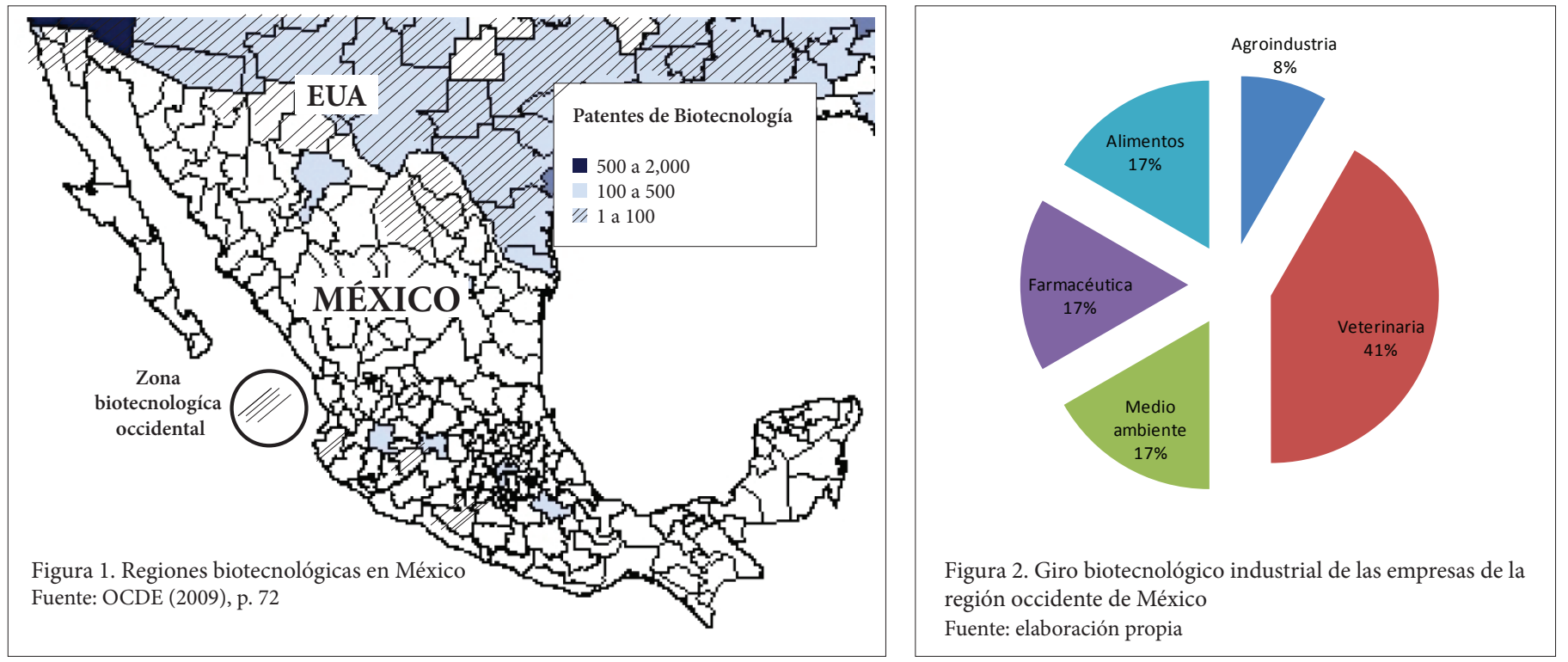

\begin{tabular}{|c|c|c|c|c|c|c|}
\hline \multirow{2}{*}{$\begin{array}{l}\text { Nombre ficticio } \\
\text { de la empresa }\end{array}$} & \multirow{2}{*}{ Tamaño } & \multirow{2}{*}{ Origen } & \multirow{2}{*}{$\begin{array}{l}\text { Desde su fundación } \\
\text { su orientación fue } \\
\text { biotecnológica }\end{array}$} & \multirow{2}{*}{$\begin{array}{c}\text { Departamento } \\
\text { de ID }\end{array}$} & \multicolumn{2}{|c|}{ Mercado atendido } \\
\hline & & & & & Nal. & Internal. \\
\hline Abiotec & Pequeña & Mexicana & & Sí & $\mathrm{X}$ & \\
\hline Bebiotec & Pequeña & Mexicana & $\mathrm{X}$ & No & $\mathrm{X}$ & $\mathrm{X}$ \\
\hline Cebiotec & Pequeña & Mexicana & $\mathrm{X}$ & Sí & $\mathrm{X}$ & $\mathrm{x}$ \\
\hline Debiotec & Micro & Mexicana & $\mathrm{X}$ & No & $\mathrm{X}$ & \\
\hline Ebiotec & Grande & Mexicana & & Sí & $\mathrm{X}$ & $\mathrm{X}$ \\
\hline Febiotec & Grande & Mexicana & & Sí & $\mathrm{x}$ & \\
\hline \multirow[t]{6}{*}{ Gebiotec } & Pequeña & Mexicana & $\mathrm{X}$ & No & $\mathrm{X}$ & \\
\hline & & Mexicana & & & & \\
\hline & & Extranjera & & & & \\
\hline & & Mexicana & & & & \\
\hline & & Mexicana & & & & \\
\hline & Pequeña & Mexicana & & & & \\
\hline
\end{tabular}

Fuente: elaboración propia.

Nota: en gris se muestra las empresas que no participaron en el estudio.

El criterio aplicado para definir el tamaño de la empresa fue el número de personal ocupado, dado por la Secretaría de Economía de México (Publicado en el Diario Oficial de la Federación el 30 de junio del 2009), donde la clasificación es la siguiente: microempresa: 0-10 empleados; pequeña empresa: 11-50 empleados; mediana empresa: 51-250 empleados.

Tabla 1. Caracterización de las empresas biotecnológicas localizadas en la región occidente de México 
El presente estudio es de corte cualitativo, exploratorio, aplicado a las empresas biotecnológicas que se logró contactar y que accedieron a participar en la investigación, en total siete, correspondiente al 58 por ciento. Se aplicaron entrevistas a profundidad, semiestructuradas - en febrero-marzo del 2013 -, al director o al encargado del área biotecnológica de las empresas participantes, enfocadas en conocer las fuentes de conocimiento externo así como detalles respecto al proceso de la búsqueda del conocimiento.

Las entrevistas se transcribieron para, a través de un análisis de contenido, identificar: la entidad (universidad, centro de investigación, instituto, e incluso empresa) a la que acuden cuando requieren de conocimiento, el tipo de relación (formal/informal) que establecen, y el tipo de conocimiento (científico o complementario) requerido. Asimismo el análisis de contenido permitió identificar detalles del proceso de búsqueda del conocimiento, tales como los factores que pueden llegar a promover u obstaculizar la formación de vínculo para acceder al conocimiento externo.

Respecto a la movilidad se preguntó acerca del tipo de industria de procedencia del entrevistado; se considera que hay movilidad si anteriormente laboró en una empresa biotecnológica.

\section{Resultados}

Los resultados muestran que las empresas cuentan con una serie de vínculos, principalmente formales, para obtener el conocimiento. También cuentan con vínculos informales aunque en mucho menor medida. Mientras que la movilidad laboral es casi nula, no obstante, sí se vislumbran indicios de este fenómeno.

Los resultados se muestran en el siguiente orden, primero se presenta el panorama total de los lazos (a todos los niveles) para la obtención de conocimiento externo para enseguida mostrar los lazos en cada uno de los niveles: regional, nacional e internacional. A continuación, se aborda el proceso que siguen las empresas para la obtención del conocimiento, iniciando en el plano regional, incluyendo la problemática que enfrenta la empresa. Luego, se aborda lo concerniente al proceso de búsqueda a nivel nacional e internacional, identificando algunos factores que inciden directamente en la realización de los vínculos.

En la figura 3 se muestra el total de los lazos identificados, en las empresas bajo estudio, para la obtención de conocimiento biotecnológico y complementario a través de relaciones formales e informales. Se aprecia un nutrido número de lazos.

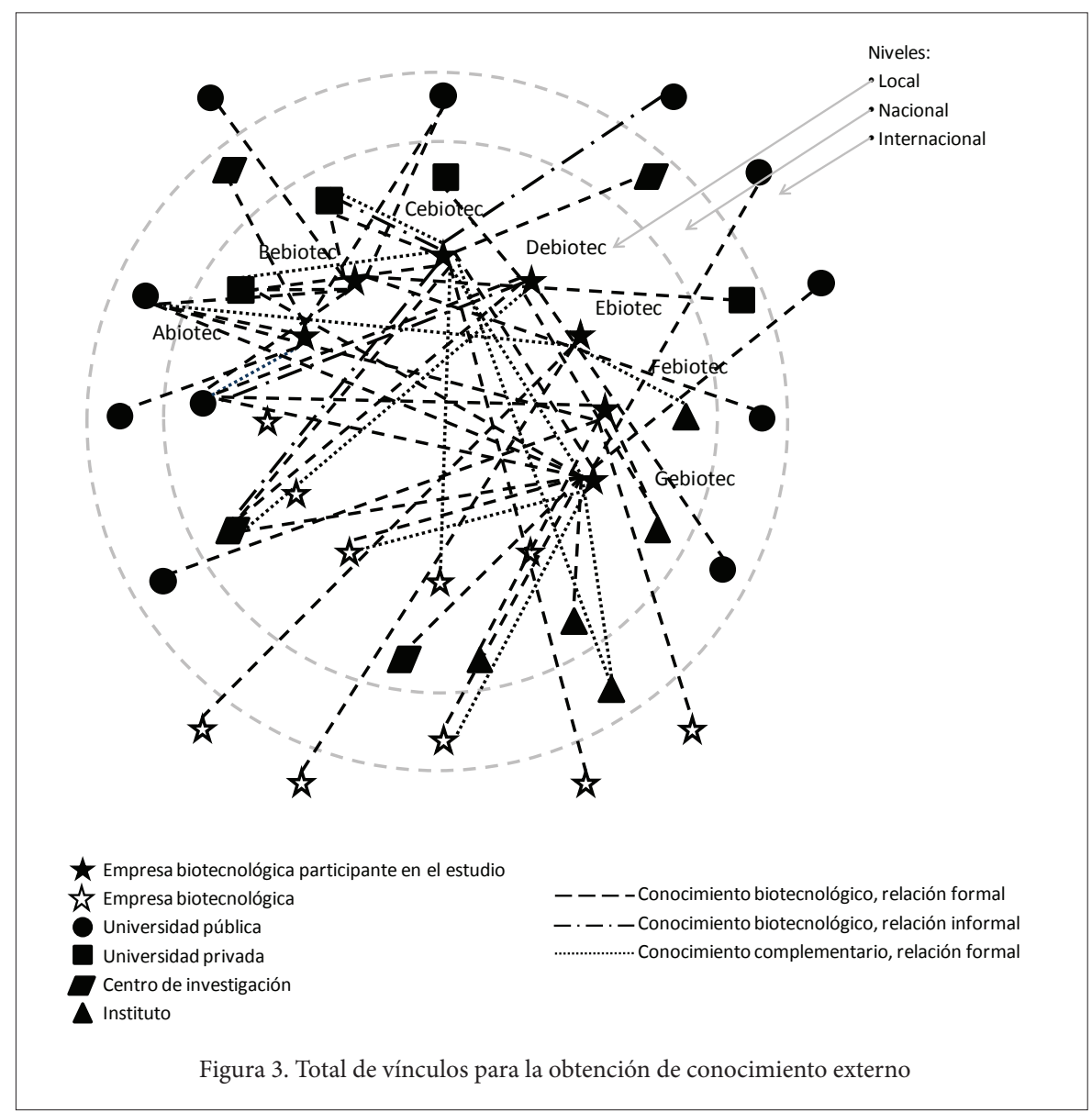

ISSN: 0718-2724. (http://jotmi.org) 
El resultado de los lazos a nivel regional se muestran en la figura 4. Si bien, aparecen en escena tres universidades privadas, el papel más importante como fuente de conocimiento corre a cargo de un centro de investigación y de una universidad pública. Los vínculos de empresa a empresa para obtener conocimiento son escasos.

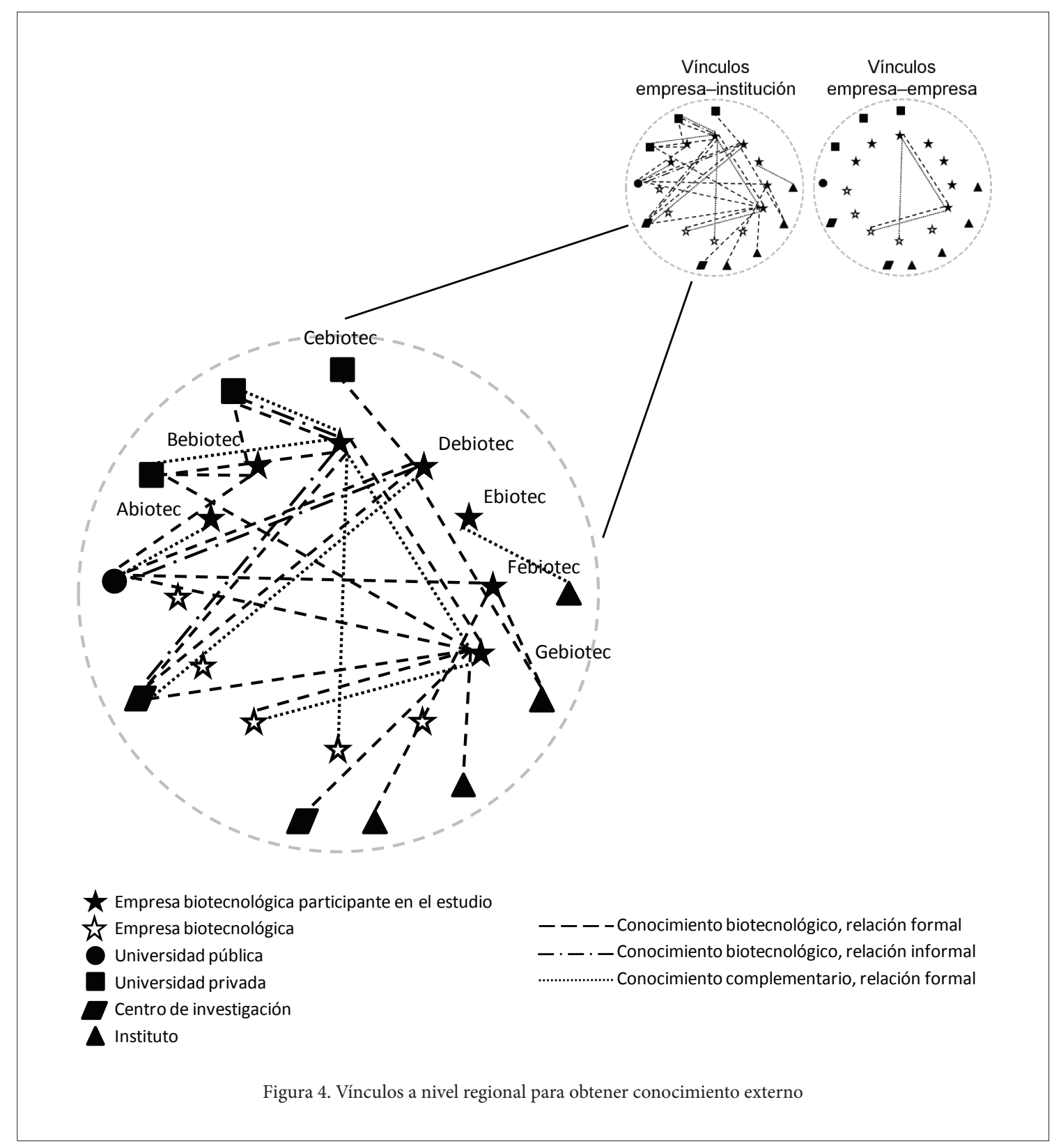

La figura 5 presenta los lazos para la obtención de conocimiento a nivel nacional e internacional. A nivel nacional (comparado con el nivel regional) se aprecia una mayor diversidad de universidades y centros de investigación. A nivel internacional (a diferencia de los niveles regional y nacional) no todas las empresas establecen lazos, tal es el caso de las empresas Abiotec y Debiotec, correspondientes a una empresa pequeña y micro, respectivamente; como fuente de conocimiento destaca la presencia de empresas y universidades en la misma proporción. 


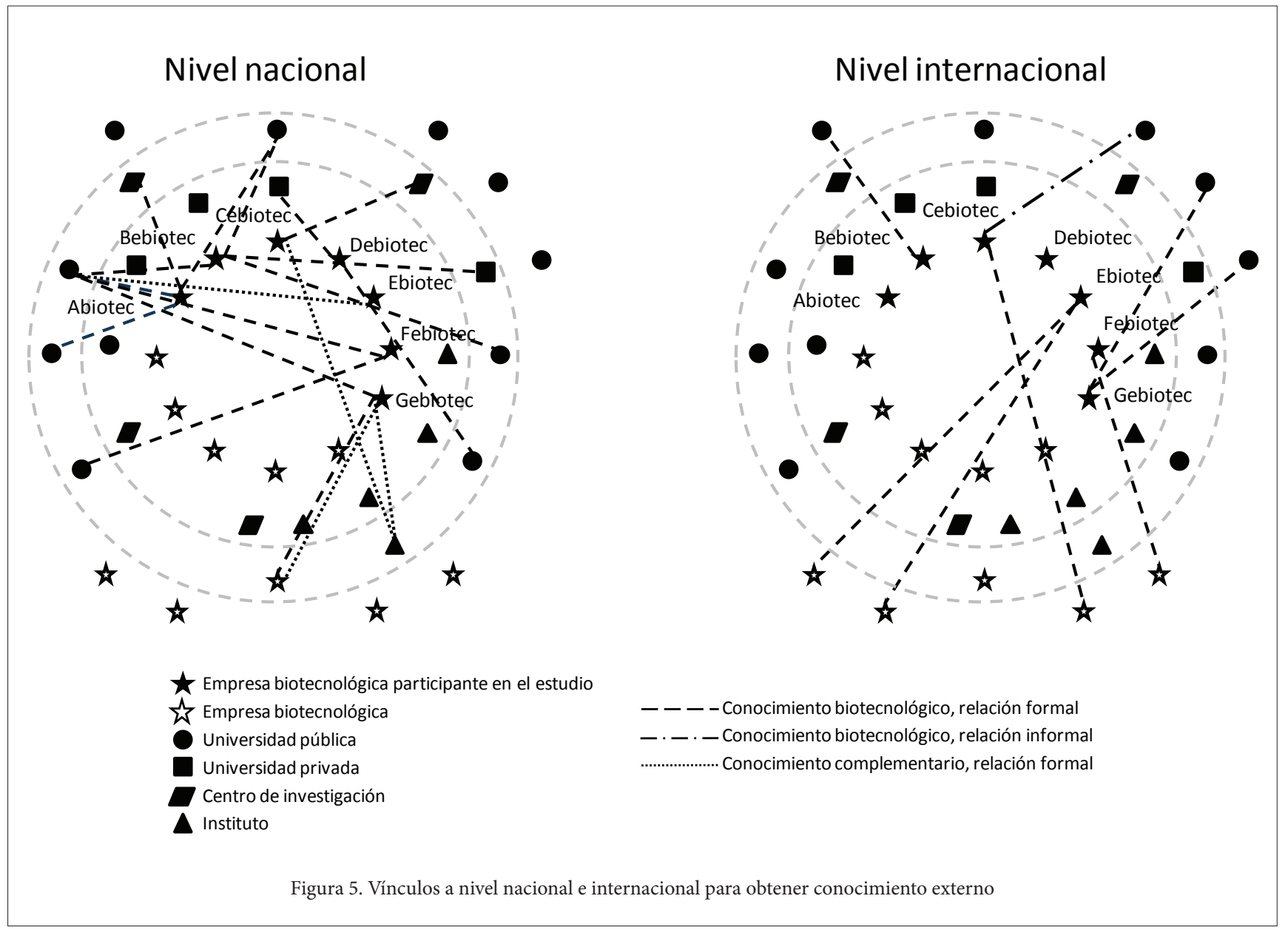

En los tres niveles (regional, nacional e internacional) predomina la relación formal para la búsqueda de conocimiento biotecnológico. La tabla 2 muestra el porcentaje de vínculos para la obtención de conocimiento.

\begin{tabular}{lcc}
\hline Nivel & Conocimiento biotecnológico & Conocimiento complementario \\
\hline Regional & $40 \%$ & $14 \%$ \\
Nacional & $25 \%$ & $7 \%$ \\
Internacional & $14 \%$ & \\
\hline
\end{tabular}

Tabla 2. Porcentaje de lazos para la obtención de conocimiento

Con respecto a la movilidad los resultados arrojan que se ha dado poco, contabilizando sólo dos casos. Enseguida se aborda el proceso de adquisición de conocimiento partiendo del nivel regional, incluyendo los obstáculos que impiden la formación del lazo.

Las empresas PYMES que no cuentan con departamento de investigación y desarrollo (ID) tienen su principal apoyo a nivel regional en universidades, centros de investigación e institutos. Lo anterior no aplica para las PYMES con departamento de ID y las grandes empresas, que acuden a las instituciones regionales sólo ocasionalmente.

Los intentos de las empresas por formar lazos a nivel regional no siempre son exitosos debido a que las instituciones presentan diversas 
deficiencias, deliberados o no, que dificultan o impiden su formación, como: 1) Niveles por debajo de sus requerimientos. Por ejemplo, algunas empresas consideran que las universidades no tienen un perfil enfocado a la industria o no están certificadas para ofrecer servicios de tercerías ${ }^{7}$. 2) Burocracia, que impide los acuerdos contractuales. Para algunos empresarios esta burocracia fomenta la colaboración informal porque "hacer un convenio es casi imposible". 3) Las propias instituciones evitan vincularse con PyMES. Por ejemplo, en el caso de tener una patente la ceden a una empresa grande.

En cuanto a la vinculación entre empresas sí existe aunque es escasa (ver figura 4). La tendencia es a no vincularse, incluso algunas empresas no tienen contemplada esta opción. Otra causa manifiesta es el hermetismo que prevalece al ser empresas competidoras.

Así como hay obstáculos para la formación de vínculos a nivel regional también hay factores que incentivan la formación de los lazos a nivel nacional e internacional. En esta investigación identificamos dos factores: el mercado y la proactividad.

Dado que el mercado es muy reñido, no sólo porque la empresa atiende el mercado internacional sino por la competencia a la que se enfrenta a nivel nacional, las empresas se dan a la tarea de ofrecer productos competitivos. Para ello, al no encontrar el conocimiento necesario dentro del país, las empresas buscan el conocimiento o conocimiento incorporado en una tecnología en el extranjero. Así, han establecido vínculos en Estados Unidos, Canadá, América del sur, Europa. El segundo factor, la personalidad proactiva ${ }^{8}$ del empresario, se manifiesta en la iniciativa de forjar lazos más densos a nivel nacional e internacional.

La mayoría de las empresas tiene una deficiencia de conocimiento complementario. Esto se debe a que las empresas surgieron de emprendedores con una formación educativa técnica, de la que precisamente partieron para fundar la empresa. Los empresarios manifestaron tener deficiencias en rubros como: mercado, protección de la propiedad industrial, administración, cuestiones fiscales.

\section{Discusión}

El presente estudio intentó identificar cuáles son los mecanismos de conocimiento externo que utilizan las empresas biotecnológicas de la región occidente de México. Se encontró que el mecanismo más utilizado es la relación formal para la búsqueda de conocimiento científico biotecnológico.
Respecto a la movilidad los resultados arrojaron que es escasa, entre los motivos están los siguientes: $i$ ) hay empresas familiares o constituida por varios socios los cuales tienen incentivos para permanecer en la firma, no para moverse a otra empresa; ii) la industria está compuesta por menos de 15 empresas biotecnológicas de diversos giros, por tanto, la oferta para moverse a una empresa similar es reducida; iii) se observa una marcada tendencia a permanecer en una empresa con intención de tener estabilidad laboral, dadas las pocas oportunidades que presenta la región.

Los procesos que siguen las empresas para la obtención del conocimiento externo distan de ser homogéneos. Aun cuando las empresas están bajo el mismo entorno y región, sus procesos pueden verse afectados por factores tales como: $i$ ) los recursos, por ejemplo, si tienen o no departamento de ID; ii) la personalidad proactiva del emprendedor, que lo lleve a explorar más allá del entorno regional, es decir, a nivel nacional o incluso internacional; iii) un mercado muy competido, debido a que se comparte el mercado con empresas connacionales y trasnacionales en territorio nacional, o porque se atiende el mercado internacional.

El análisis muestra que las empresas exploran o buscan por varias vías hasta encontrar el conocimiento que requieren, esto es, las redes para acceder al conocimiento no están construidas del todo, sino que se van formando a medida que surgen las necesidades de conocimiento, pero también los obstáculos (por ejemplo, la burocracia o el bajo nivel científico de las universidades).

La iniciativa de establecer vínculos más densos, y a nivel internacional, está relacionada no sólo con el crecimiento de la empresa sino también con su participación en el mercado internacional. Lo que sugiere que el conocimiento adquirido a nivel internacional confiere a la empresa una competitividad de clase mundial.

Con lo anterior, se espera haber atendiendo la sugerencia de autores como Breschi y Catalini de realizar "estudios cualitativos enfocados a capturar las sutilezas involucradas en las interacciones" (2010, p. 25), en este caso, el acceso a conocimiento.

Los resultados reportados tienen algunas implicaciones de política pública. Primero, al ser las universidades y centros de investigación públicos fuente de conocimiento deben ser menos burocráticos, de manera que incentiven el acercamiento de las empresas; este punto es crucial toda vez que los resultados muestran que el lazo predominante es de tipo formal. Segundo, las universidades y

(7) Se denomina tercería cuando se acude a un tercero por: 1) cuestión normativa, por ejemplo, la realización de estudios preclínicos; 2) realización de pruebas especiales. En el primer caso se acude a una instancia certificada. En el segundo caso a una empresa o institución que cuente con los medios y recursos para llevar a cabo la prueba en cuestión.

(8) La personalidad proactiva para Bateman y Crant es aquella que está relativamente libre de fuerzas situacionales, mientras que los individuos que no tienen esta características son relativamente pasivos, es decir, reaccionan, se adaptan y terminan moldeados por el entorno. La gente proactiva explora en busca de oportunidades, muestra iniciativa, se pone en acción, y persevera hasta que logra el cambio deseado; la personalidad opuesta, no identifica y muchos menos aprovecha las oportunidades para cambiar las cosas, tiene poca iniciativa y espera a que otros hagan los cambios, pasivamente se adaptan y perpetúan el estado de su entorno (1993). Para Crant la proactividad es "tomar la iniciativa para mejorar las circunstancias actuales o crear unas nuevas; esto implica desafiar el status quo y no adaptarse pasivamente a las condiciones actuales” (2000, p. 436). 
centros de investigación públicos deben elevar su nivel a fin de poder atender las demandas de las empresas de conocimiento científico biotecnológico, sin olvidar los requerimientos de conocimiento complementario, como: manufactura, mercado, administrativo, propios de cualquier empresa. Por último, y no menos importante, es necesario fomentar a través de instituciones públicas la vinculación con entidades relevantes en el plano internacional por ser ahí donde se genera el conocimiento.

El estudio tiene varias limitaciones. Primero, muestra los resultados específicos de una región de un país en desarrollo por lo que los resultados no se prestan a hacer generalizaciones. Sin embargo, sí permite un acercamiento a la comprensión de la manera en que las empresas obtienen el conocimiento externo, los factores que pueden obstaculizarlo y aquellos que le dan impulso. Segundo, la reducida población de empresas de biotecnología de la región bajo estudio no permite todavía el surgimiento de algunos fénomenos propios de aglomeraciones más nutridas, tal es el caso de la movilidad. Tercero, las entrevistas estuvieron limitadas a la apertura del entrevistado ya que el empresario de la región bajo estudio suele ser muy reservado.

\section{Referencias}

Audretsch, D. y Feldman, M. (2003). Small-Firm Strategic Research Partnerships: The Case of Biotechnology. Technology Analysis and Strategic Management, 15(2), 273-288.

Balzat, M. y Hanusch, H. (2004). Recent Trends in the Research in National Innovation Systems. Journal of Evolutionary Economics, 14, 197-210.

Bateman, T. S. y Crant, J. M. (1993). The Proactive Component of Organizational Behavior: A Measure and Correlates. Journal of Organizational Behavior, 14(2), 103-118.

Breschi, S. y Catalini, C. (2010). Tracing the Links between Science and Technology: An Exploratory Analysis of Scientists' and Inventors' Networks. Research Policy, 39(1), 14-26.

Carayannopoulos, S. y Auster, E. R. (2010). External Knowledge Sourcing in Biotechnology through Acquisition versus Alliance: A KBV Approach. Research Policy, 39(2), 254-267.

Chaminade, C. y Edquist, C. (2005). From Theory to Practice: The Use of Systems of Innovation Approach in Innovation Policy. Centre for Innovation, Research and Competence in the Learning Economy, Lund University. Manuscrito en preparación.

Crant, J. M. (2000). Proactive Behavior in Organizations. Journal of Management, 26(3), 435-462.

Decarolis, D. M. y Deeds, D. L. (1999). The Impact of Stocks and Flows of Organizational Knowledge on Firm Performance: An Empirical Investigation of the Biotechnology Industry. Strategic Management Journal, 20(10), 953-968.
Doloreux, D. (2002). What we should know about Regional Systems of Innovation. Technology in Society, 24(3), 243-263.

Doloreux, D. y Parto, S. (2005). Regional Innovation Systems: Current Discourse and Unresolved Issues. Technology in Society, 27(2), 133153.

Edgington, D. W. (2008). The Japanese Innovation System: UniversityIndustry Linkages, Small Firms and Regional Technology Clusters. Prometheus, 26(1), 1-19.

Fabrizio, K. R. (2009). Absorptive Capacity and the Search for Innovation. Research Policy, 38(2), 255-267.

Granstrand, O. (1998). Towards a Theory of the Technology-Based Firm. Research Policy, 27(5), 465-489.

Griliches, Z. (1990). Patent Statistics as Economic Indicators: A Survey. Journal of Economic Literature, 28(4), 1661-1707.

Haeussler, C., Patzelt, H. y Zahra, S. A. (2012). Strategic Alliances and Product Development in High Technology New Firms: The Moderating Effect of Technological Capabilities. Journal of Business Venturing, 27(2), 217-233.

Khoury, T. A. y Pleggenkuhle, E. G. (2011). Shared Inventions and the Evolution of Capabilities: Examining the Biotechnology Industry. Research Policy, 40(7), 943-956.

Larsson, R., Bengtsson, L., Henriksson, K. y Sparks, J. (1998). The Interorganizational Learning Dilemma: Collective Knowledge Development in Strategic Alliances. Organization Science, 9(3), 285-305.

Lawton-Smith, H. y Waters, R. (2005). Employment Mobility in High-Technology Agglomerations: The Cases of Oxfordshire and Cambridgeshire. Area, 37(2), 189-198.

Maliranta, M., Mohnen, P. y Rouvinen, P. (2009). Is Inter-firm Labor Mobility a Channel of Knowledge Spillovers? Evidence from a Linked Employer-Employee Panel. Industrial and Corporate Change, 18(6), 1161-1191.

Moen, J. (2001). Is mobility of Technical Personnel a Source of R\&D Spillovers? Department of Economics Discussion Paper 05/01, Norwegian School of Economics and Business Administration.

OCDE (2009). Biotechnology Statistics 2009. OCDE, París.

Owen-Smith, J. y Powell, W. W. (2004). The Effects of Spillovers in the Boston Biotechnology Community. Organization Science, 15(1), 5-21.

Pisano, G. P. (1994). Knowledge, Integration, and the Locus of Learning: An Empirical Analysis of Process Development. Strategic Management Journal, 15, 85-100. 
Porter, J., Oliver, A., Zucker, L. y Brewer, M. (1996). Social Networks, Learning, and Flexibility: Sourcing Scientific Knowledge in New Biotechnology Firms. Organization Science, 7(4), 428-443.

Powell, W. W. (1998). Learning from Collaboration: Knowledge and Networks in the Biotechnology and Pharmaceutical Industries. California Management Review, 40(3), 228-240.

Powell, W. W., Koput, K. W. y Smith, L. (1996). Interorganizational Collaboration and the Locus of Innovation: Networks of Learning in Biotechnology. Administrative Science Quarterly, 41(1), 116-145.

Prevezer, M. (1997). The Dynamics of Industrial Clustering in Biotechnology. Small Business Economics, 9(3), 255-271.

Rank, C., Rank, O. y Wald, A. (2006). Integrated Versus CorePeriphery Structures in Regional Biotechnolgy Networks. European Management Journal, 24(1), 73-85.
Salavisa, I., Sousa, C. y Fontes M. (2012). Topologies of Innovation Networks in Knowledge-intensive Sectors: Sectoral Differences in the Access to Knowledge and Complementary Assets through Formal and Informal Ties. Technovation, 32(6), 380-399.

Smith, K. (2000). Innovation as a Systemic Phenomenon: Rethinking the Role of Policy. Enterprise and Innovation Management Studies, 1(1), 73-102.

Song, J., Almeida, P. y Wu, G. (2003). When is Mobility more likely to Facilitate Interfirm Knowledge Transfer? Management Science, 49(4), 351-365.

Tzabbar, D., Aharonson, B. S. y Amburgey, T. L. (2013). When does Tapping External Sources of Knowledge Result in Knowledge Integration? Research Policy, 42(2), 481-494. 